\title{
Positional installation of contrast (PIC) and Redo-PIC cystography for diagnosis of occult vesicoureteral reflux
}

\author{
Kıvilcım Karadeniz-Cerit ${ }^{1}$, David Terence Thomas ${ }^{2}$, Raziye Ergun ${ }^{3}$, Nurdan Yildiz ${ }^{4}$, \\ Harika Alpay ${ }^{4}$ Sabahat İnanir ${ }^{5}$, E. Tolga Dağl1 ${ }^{3}$, Halil Tuğtepe ${ }^{1,3}$ \\ Division of ${ }^{1}$ Pediatric Surgery, ${ }^{3}$ Pediatric Urology and ${ }^{4}$ Pediatric Nephrology, Department of Pediatrics, Department of \\ ${ }^{5}$ Nuclear Medicine, Marmara University School of Medicine, ${ }^{2}$ Department of Pediatric Surgery, Maltepe University Faculty \\ of Medicine Istanbul, Turkey. E-mail: htugtepe@yahoo.com \\ Received: 24th July 2017, Revised: 25th September 2017, Accepted: 30th October 2017
}

SUMMARY: Karadeniz-Cerit K, Thomas DT, Ergun R, Yıldız N, Alpay H, İnanır $S$, Dağlı ET, Tuğtepe H. Positional installation of contrast (PIC) and RedoPIC cystography for diagnosis of occult vesicoureteral reflux. Turk J Pediatr 2018; 60: 180-187.

To evaluate the value of Positional Installation of Contrast (PIC) and RedoPIC cystography in patients with febrile recurrent urinary tract infection (f-UTI) where voiding cystourethrogram (VCUG) was negative. Patients with recurrent f-UTI with no reflux on VCUG referred to the outpatient clinic of Pediatric Urology, between June 2011 and June 2016 were included in the study. A PIC cystography was performed in all patients. When reflux was found, subureteric injection was performed. Urinary cultures were used for follow-up. Patients that continued having f-UTI, received redo-PIC cystography. PIC cystography was performed on 42 patients. The average age of patients was 8.0 \pm 3.6 years. Vesicoureteral reflux (VUR) was detected in 41 patients. Average follow-up time after PIC cystography was 44.6 months. Thirty-three patients (80.5\%) were free of f-UTI after PIC cystography and concurrent subureteric injection. Eight patients continued to have recurrent f-UTI. Six of these patients underwent redo-PIC cystography and PIC-VUR was demonstrated in all patients. After an average follow up of 30.9 months, no f-UTI was seen in these patients. The success rate of $80.5 \%(33 / 41)$ after 1st PIC cystography and subureteric injection increased to $95.1 \%(39 / 41)$ after redo-PIC cystography in six patients. Patients with recurrent f-UTIs without VUR on VCUG are an important challenge. PIC cystography is an important tool in demonstrating occult VUR in these patients. We advise that PIC cystography is performed in all patients with recurrent f-UTI with negative VCUG and redo-PIC cystography in patients who continue to have f-UTI after 1st PIC cystography and subureteric injection.

Key words: Positional instillation of contrast cystography, vesico-ureteral reflux, urinary tract infection, cystoscopy, occult VUR.

Febrile-UTI (f-UTI) is most commonly associated with vesicoureteral reflux (VUR), and it plays an important role in the formation of renal parenchymal lesions and scars leading to problems such as hypertension, chronic renal failure and end stage renal disease. ${ }^{1-6}$ At the time of diagnosis, $30-49 \%$ of children with VUR are reported to have renal parenchymal scarring. ${ }^{2,7}$ Therefore, guidelines for the management of f-UTI have advocated aggressive treatment and extensive imaging studies to detect VUR and renal scarring. 4,5,8
Voiding cystourethrogram (VCUG) is considered the standard method of evaluation to identify VUR in children with recurrent $\mathrm{f}$-UTI. When a standard VCUG does not show VUR in children who have experienced recurrent f-UTI, clinical management is controversial. Rubenstein et al. ${ }^{9}$ introduced a novel cystogram technique to evaluate the competence of the ureteral valve mechanism. There are several studies reporting the use of positional instillation of contrast (PIC) cystography in patients with recurrent f-UTI but no VUR on conventional VCUG. PIC 
cystography has the advantage of the ability to perform subureteric injection in patients who are found to have occult vesicoureteral reflux (PIC-VUR) in the same session. To our knowledge, there are no studies reporting the use of redo-PIC cystography in patients

Table I. PIC-VUR Grade and Follow-up Findings of the Patients with Normal USG and DMSA Finding.

\begin{tabular}{|c|c|c|c|}
\hline Patient no & Kidney & PIC-VUR grade & Follow-up after 1st SUI \\
\hline \multirow[t]{2}{*}{2} & $L K$ & 0 & No recurrent f-UTI \\
\hline & $R K$ & 2 & \\
\hline \multirow[t]{2}{*}{3} & $L K$ & 2 & No recurrent f-UTI \\
\hline & $R K$ & 1 & \\
\hline \multirow[t]{2}{*}{5} & $L K$ & 1 & No recurrent f-UTI \\
\hline & $R K$ & 2 & \\
\hline \multirow[t]{2}{*}{8} & $L K$ & 3 & Recurrent f-UTI \\
\hline & $R K$ & 2 & \\
\hline \multirow[t]{2}{*}{9} & $L K$ & 2 & No recurrent f-UTI \\
\hline & $R K$ & 2 & \\
\hline \multirow[t]{2}{*}{12} & $L K$ & 1 & No recurrent f-UTI \\
\hline & $R K$ & 2 & \\
\hline \multirow[t]{2}{*}{13} & $L K$ & 2 & Recurrent f-UTI \\
\hline & $R K$ & 0 & \\
\hline \multirow[t]{2}{*}{14} & $L K$ & 2 & No recurrent f-UTI \\
\hline & $R K$ & 0 & \\
\hline \multirow[t]{2}{*}{15} & $L K$ & 1 & No recurrent f-UTI \\
\hline & $R K$ & 1 & \\
\hline \multirow[t]{2}{*}{16} & $L K$ & 1 & No recurrent f-UTI \\
\hline & $R K$ & 1 & \\
\hline \multirow[t]{2}{*}{18} & $L K$ & 1 & No recurrent f-UTI \\
\hline & $R K$ & 1 & \\
\hline \multirow[t]{2}{*}{20} & $L K$ & 1 & No recurrent f-UTI \\
\hline & $R K$ & 1 & \\
\hline \multirow[t]{2}{*}{21} & $L K$ & 2 & No recurrent f-UTI \\
\hline & $R K$ & 0 & \\
\hline \multirow[t]{2}{*}{22} & $L K$ & 2 & Recurrent f-UTI \\
\hline & $R K$ & 0 & \\
\hline \multirow[t]{2}{*}{24} & $L K$ & 0 & Recurrent f-UTI \\
\hline & $R K$ & 2 & \\
\hline \multirow[t]{2}{*}{25} & $L K$ & 2 & No recurrent f-UTI \\
\hline & $R K$ & 2 & \\
\hline \multirow[t]{2}{*}{30} & $L K$ & 2 & No recurrent f-UTI \\
\hline & $R K$ & 2 & \\
\hline \multirow[t]{2}{*}{31} & $L K$ & 2 & No recurrent f-UTI \\
\hline & $R K$ & 1 & \\
\hline \multirow[t]{2}{*}{39} & $L K$ & 2 & No recurrent f-UTI \\
\hline & $R K$ & 1 & \\
\hline \multirow[t]{2}{*}{40} & $L K$ & 1 & No recurrent f-UTI \\
\hline & $R K$ & 1 & \\
\hline
\end{tabular}


who continue to have f-UTI after subureteric injection for PIC-VUR.

The aim of this study is to evaluate the use of PIC cystography and to present our experience with redo-PIC cystography, that has not been previously reported in literature.

\section{Material and Methods}

Patients with recurrent f-UTI with no reflux on VCUG referred to the outpatient clinic of Pediatric Urology, between June 2011 and June 2016 were included in the study and the data of the patients was analyzed retrospectively. The study was approved by the Ethics Committee (09.2017.288).

Patients applying to our outpatient clinic with recurrent f-UTI were evaluated according to the standard protocol of our department. This evaluation included medical history, voiding diary, uroflowmetry, urinary ultrasonography, Tc99m dimercaptosuccinic acid (DMSA) scan and voiding cystourethrography (VCUG). Patients were commenced on prophylactic antibiotics and those with constipation were always treated. Patients with vesicoureteral reflux, dysfunctional elimination or dysfunctional voiding and anatomical pathologies were excluded from this study. Recruitment flowchart for this study is shown in Figure 1. Follow-up was conducted until September 2016.

Urinary USG evaluated renal units for hydronephrosis, pelvicaliectasis and ureteral dilatation. Tc-99m DMSA evaluated for renal scarring, hypoactivity, functional loss, pelvicaliectasis and findings of pyelonephritis. Uroflow and voiding diaries were utilized to eliminate the diagnosis of dysfunction voiding. Patients with staccato pattern or fractionated voiding with electromyogram (EMG) activity on at least two uroflow studies were considered as dysfunctional voiding, according to ICCS guidelines. ${ }^{10}$ All patients either received trimethoprim/sulfamethoxazole or nitrofurantoin as a prophylactic antibiotic. Recurrent UTI was defined as either two or more episodes of UTI with acute pyelonephritis/ upper urinary tract infection, or; one episode of UTI with acute pyelonephritis/upper urinary tract infection plus one or more episode of UTI with cystitis/lower urinary tract infection, or; three or more episodes of UTI with cystitis/ lower urinary tract infection, as defined in
NICE Clinical Guideline 54.11 Constipation was diagnosed as patients having type 1 or 2 stools according to the Bristol Stool Scale, and if present was always treated, with osmotic agents plus advice of dietary changes. f-UTI was defined as $>10^{6} \mathrm{cfu}$ colonies at culture (clean stream urine) plus associated fever with or without urinary tract symptoms. Fever was defined as an axillary temperature above $38^{\circ}$ Celsius, confirmed at hospital setting.

The study group included all patients with recurrent f-UTI and no VUR on VCUG. To limit the potential confounding variables, patients with dysfunctional elimination diagnosed through history (urgency, frequency, constipation, etc.) or established by uroflow plus voiding diary and patients with other anatomical abnormalities causing f-UTI (duplication, diverticulae, ureteropelvic junction obstruction, ureterocele, etc) were excluded from the study.

After informed consent, PIC cystography was performed in all patients of the study group. The technique of PIC cystography performed at the time of cystoscopy was as described previously by Rubenstein ${ }^{9}$. The technique of PIC cystography performed at the time of cystoscopy was as follows:

Routine rigid cystoscopy is performed to evaluate for anatomical abnormalities

The bladder is emptied

The cystoscope beak is positioned to face the urethral orifice, close enough that the cystoscopic view is filled by the ureteral orifice but not inside it

Contrast is placed $1 \mathrm{~m}$ above the level of the bladder

Contrast is flowed toward the ureteral orifice through the irrigation port of the cystoscope while fluoroscopy is performed

The bladder is emptied and the procedure is repeated on the contralateral side.

Subureteric injection was subsequently performed on the patients in whom PICVUR was identified. An injection needle was advanced through the operating channel of the cystoscope to approach the ureteric orifice at the 6 o'clock position. The needle was advanced in the plane between the bladder mucosa and bladder muscle. The injection was performed 
Table II. PIC-VUR Grade and Follow-up Findings of the Patients with Abnormal USG and DMSA Finding

\begin{tabular}{|c|c|c|c|c|c|}
\hline $\begin{array}{l}\text { Patient } \\
\text { no }\end{array}$ & Kidney & USG & DMSA & $\begin{array}{l}\text { PIC-VUR } \\
\text { grade }\end{array}$ & $\begin{array}{l}\text { Follow-up after } \\
\text { 1st SUI }\end{array}$ \\
\hline \multirow[t]{2}{*}{1} & LK & Pelvicalyseal dilatation & $\begin{array}{l}\text { Paranchymal scarring } \\
\text { \& Pelvicalyseal } \\
\text { dilatation }\end{array}$ & 0 & \multirow[t]{2}{*}{$\begin{array}{l}\text { No recurrent } \\
\text { f-UTI }\end{array}$} \\
\hline & RK & Normal & Normal & 2 & \\
\hline \multirow[t]{2}{*}{4} & LK & Normal & Normal & 0 & \multirow[t]{2}{*}{ Recurrent f-UTI } \\
\hline & RK & Normal & Paranchymal scarring & 2 & \\
\hline \multirow[t]{2}{*}{6} & LK & Pelvicalyectasia & $\begin{array}{l}\text { at lower pole } \\
\text { Normal }\end{array}$ & 2 & \multirow[t]{2}{*}{ Recurrent f-UTI } \\
\hline & RK & Pelvicalyectasia & Normal & 2 & \\
\hline \multirow[t]{2}{*}{7} & LK & Pelvicalyseal dilatation & Dilatation & 1 & \multirow{2}{*}{$\begin{array}{l}\text { No recurrent } \\
\text { f-UTI }\end{array}$} \\
\hline & RK & Normal & Normal & 0 & \\
\hline \multirow[t]{2}{*}{10} & LK & Hypoplasia & Parenchymal scarring & 1 & \multirow{2}{*}{$\begin{array}{l}\text { No recurrent } \\
\text { f-UTI }\end{array}$} \\
\hline & RK & Normal & Normal & 2 & \\
\hline \multirow[t]{2}{*}{11} & LK & Normal & Acute pyelonephritis & 0 & \multirow[t]{2}{*}{ Lost to Follow-up } \\
\hline & RK & Normal & Normal & 0 & \\
\hline \multirow[t]{2}{*}{17} & LK & Normal & Parenchymal scarring & 0 & \multirow{2}{*}{$\begin{array}{l}\text { No recurrent } \\
\text { f-UTI }\end{array}$} \\
\hline & RK & Normal & Normal & 2 & \\
\hline \multirow[t]{2}{*}{19} & LK & Normal & Paranchymal scarring & 0 & \multirow{2}{*}{$\begin{array}{l}\text { No recurrent } \\
\text { f-UTI }\end{array}$} \\
\hline & RK & Normal & $\begin{array}{l}\text { at left upper pole } \\
\text { Normal }\end{array}$ & 1 & \\
\hline \multirow[t]{2}{*}{23} & LK & Normal & Paranchymal scarring & 1 & \multirow{2}{*}{$\begin{array}{l}\text { No recurrent } \\
\text { f-UTI }\end{array}$} \\
\hline & RK & Normal & $\begin{array}{l}\text { at left upper pole } \\
\text { Normal }\end{array}$ & 1 & \\
\hline \multirow[t]{2}{*}{26} & LK & Normal & Normal & 0 & \multirow{2}{*}{$\begin{array}{l}\text { No recurrent } \\
\text { f-UTI }\end{array}$} \\
\hline & RK & Normal & Paranchymal scarring & 2 & \\
\hline \multirow[t]{2}{*}{27} & LK & Normal & $\begin{array}{l}\text { at right lower pole } \\
\text { Normal }\end{array}$ & 0 & \multirow{2}{*}{$\begin{array}{l}\text { No recurrent } \\
\text { f-UTI }\end{array}$} \\
\hline & $\mathrm{RK}$ & Normal & Paranchymal scarring & 2 & \\
\hline \multirow[t]{2}{*}{28} & LK & Pelvicalyectasis & $\begin{array}{l}\text { at right lower pole } \\
\text { hypoplasia }\end{array}$ & 2 & \multirow{2}{*}{$\begin{array}{l}\text { No recurrent } \\
\text { f-UTI }\end{array}$} \\
\hline & RK & Pelvicalyectasis & Paranchymal scarring & 2 & \\
\hline 29 & LK & Normal & $\begin{array}{l}\text { at right upper pole } \\
\text { Normal }\end{array}$ & 0 & No recurrent \\
\hline & RK & Normal & Hypoactive area in & 2 & f-UTI \\
\hline 32 & LK & Pelvicalyectasis & $\begin{array}{l}\text { middle pole } \\
\text { Normal }\end{array}$ & 2 & No recurrent \\
\hline & RK & Pelvicalyectasis & Hypoactive area in & 2 & f-UTI \\
\hline 33 & LK & Normal & $\begin{array}{l}\text { middle pole } \\
\text { Normal }\end{array}$ & 0 & No recurrent \\
\hline & RK & Pelvicalyectasis & Scarring in upper & 2 & f-UTI \\
\hline 34 & LK & Normal & pole & 0 & No recurrent \\
\hline & RK & Normal & Hypoactive area in & 3 & f-UTI \\
\hline 35 & LK & Normal & $\begin{array}{l}\text { middle pole } \\
\text { Normal }\end{array}$ & 1 & No recurrent \\
\hline & RK & Normal & Hypoactive area in & 2 & f-UTI \\
\hline 36 & LK & Normal & $\begin{array}{l}\text { upper pole } \\
\text { Hypoactive area in }\end{array}$ & 2 & No recurrent \\
\hline & RK & Normal & $\begin{array}{l}\text { upper pole } \\
\text { Normal }\end{array}$ & 1 & f-UTI \\
\hline 37 & LK & Normal & Cortical defect in & 1 & Recurrent f-UTI \\
\hline & RK & Normal & $\begin{array}{l}\text { upper \& lower pole } \\
\text { Cortical defect in }\end{array}$ & 1 & \\
\hline 38 & LK & Pelvicalyectasis & $\begin{array}{l}\text { upper pole } \\
\text { Norma1 }\end{array}$ & 2 & No recurrent \\
\hline & RK & Normal & Normal & 1 & f-UTI \\
\hline 41 & LK & Pelvicalyseal dilatation & Cortical scar in upper & 1 & No recurrent \\
\hline & RK & Normal & $\begin{array}{l}\text { and lower poles } \\
\text { Normal }\end{array}$ & 1 & \\
\hline 42 & LK & Normal & Cortical scar in & 1 & Recurrent f-UTI \\
\hline & RK & Normal & $\begin{array}{l}\text { middle pole } \\
\text { Normal }\end{array}$ & 1 & \\
\hline
\end{tabular}

LK: Left Kidney, RK: Right Kidney, f-UTI: Febrile Urinary Tract Infection, PIC-VUR: Positional Installation of Contrast Cystoscopy - Vesicouretheral Reflux, 


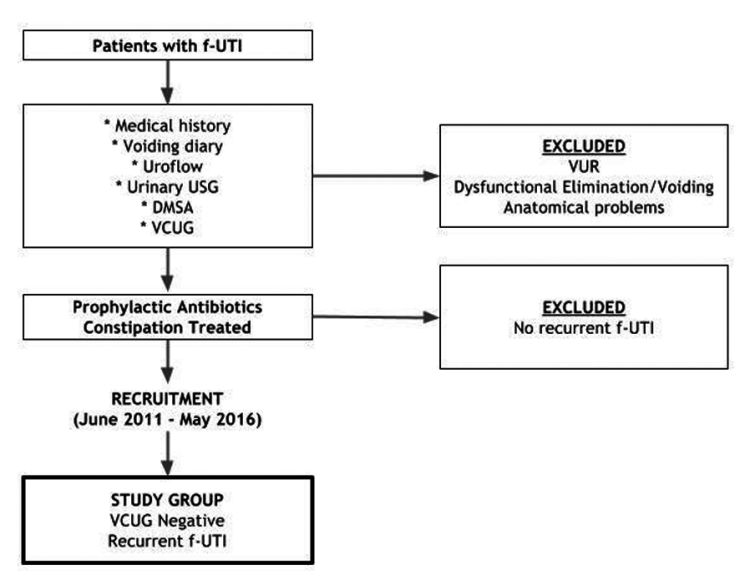

Fig. 1. Recruitment algorithm for patients included in this study.

until the appearance of the ureteric orifice resembled an inverted crescent. Patients were then followed with clinical symptoms and urinary culture. Prophylactic antibiotics were stopped at postoperative $1^{\text {st }}$ month.

During follow-up, patients had outpatient visits and urinary cultures, once per month during the $1^{\text {st }}$ year and every 3 months during $2^{\text {nd }}$ year and upon symptoms thereafter.

A subgroup of patients who continued to have recurrent f-UTI after PIC cystography and subureteric injection had a second PIC cystography performed.

Patients' age, gender, urinary USG and DMSA findings, PIC cystography results and follow-up time and presence of f-UTI during follow-up were noted.

\section{Results}

There were 39 girls and 3 boys included in this study. The average age was $8.0 \pm 3.6$ years (11 months-16 years). Patients' radiological, PIC-VUR and follow-up findings are shown in Table I-II.

Urinary USG was abnormal in eight (19.0\%) and DMSA was abnormal in $20(47.6 \%)$ of patients. There was no correlation between PIC cystography results or pathological finding on either urinary USG or DMSA.

PIC cystography demonstrated VUR in 41 children and no reflux was found in one child. PIC-VUR was bilateral in 25 patients, unilateral in 16 patients (66/84 renal units). PIC-VUR was graded as grade 1 in 30 units $(45.5 \%)$, grade 2 in 34 units $(51.5 \%)$ and

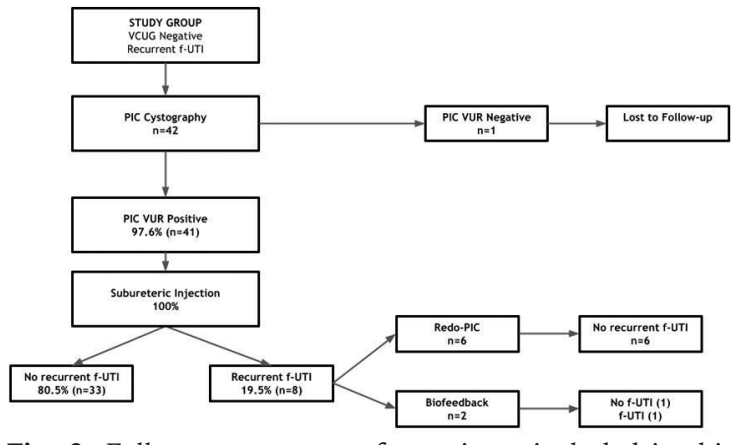

Fig. 2. Follow-up outcomes for patients included in this study.

grade 3 in 2 units (3.0\%). Subureteric injection was performed in all of the patients in whom PIC-VUR was identified. The average followup time after PIC cystography was $44.6 \pm 17.3$ months (4.5 - 64.2 months).

Thirty-three patients $(80.5 \%)$ were free of f-UTI after PIC cystogram and concurrent subureteric injection. Eight patients continued to have recurrent f-UTI. After informed consent, six patients underwent redo-PIC cystography while two patient's families refused redo-PIC cystography. PIC-VUR was demonstrated in all patients undergoing redo-PIC cystography. Radiological, PIC-VUR and follow-up findings for these patients are shown in Table I-II. After an average follow-up of 30.9 months, no f-UTI was seen in these patients. The success rate of $80.5 \%(33 / 41)$ after 1st PIC cystography and subureteric injection increased to $95.1 \%$ (39/41) after redo-PIC cystography in six patients. Follow-up findings for all patients are shown in Figure 2.

One patient who was not found to have PICVUR was lost to follow-up. Despite no evidence of dysfunctional voiding, the two patients whose parents refused redo-PIC cystography were commenced on a treatment of biofeedback, as a last resort effort to treat their f-UTI. While one patient remained UTI free after 6 months of biofeedback therapy, one patient continued to have recurrent f-UTI after biofeedback.

\section{Discussion}

VUR is caused by an insufficient valve mechanism at the ureterovesical junction, related to short submucosal length, weak detrusor support or periureteral diverticulum. ${ }^{9}$ The gold standard method to diagnose VUR is 
VCUG. This method has evolved over the years. Cases of non-VUR f-UTI/pyelonephritis are particularly important because they frequently recur. Recurrences develop in about $45 \%$ of girls and about $15 \%$ of boys (about $20 \%$ overall). ${ }^{12,13}$ This group of patients are likely associated with important morbidity such as renal damage, recurrent hospitalization, increased hospitalization costs, parental loss of time from work and frustration for the doctor and family.

Rubenstein et al. ${ }^{9}$ have shown a novel approach that cystoscopically examines and challenges the ureteral orifices in this subset of children. In 30 children with normal VCUG findings and a history of recurrent f-UTIs, PIC-VUR was diagnosed in all 30 using PIC cystography. The study included two control groups. In the first control group of 15 patients with no f-UTI and no VUR, PIC cystography did not reveal PIC-VUR. In their second control group of 15 ureters of patients with f-UTI and VUR, PIC cystography revealed PIC-VUR in all patients. They found PIC cystography was accurate in diagnosing VUR in comparison to the standard cystogram in $91 \%$ of patients.

Edmondson et al. ${ }^{3}$ evaluated the reproducibility of this finding using a prospective multiinstitutional collaborative examination to determine whether PIC-VUR is as common in such cases as previously shown. PIC-VUR was identified in $82 \%$ of the patients with f-UTIs and negative VCUGs. Tareen et al. ${ }^{5}$ performed a similar study in a small number of patients. All 5 patients in this study showed PIC-VUR and all were treated with endoscopic injection of dextranomer/hyaluronic acid copolymer or vesicoureteral reimplantation. The results of our study reveal that the incidence of occult VUR shown by PIC cystography was $97.1 \%$ with only one patient having a normal PIC cystography result.

In our study, 33 of 41 the patients $(80.5 \%)$ were free of $\mathrm{f}$-UTI after ureteric injection during our follow-up. Additionally, six patients who had a second PIC cystogram and subureteric injection had no recurrent f-UTI after an average follow-up of 30.9 months. Noe and Williams ${ }^{7}$ reported their experience with PIC cystography and simultaneous dextranomer/ hyaluronic acid copolymer injection in 47 children with pyelonephritis and negative
VCUGs. A total of $75 \%$ of the patients had PIC-VUR and were treated endoscopically. Three of the patients developed febrile f-UTI after subureteric injection and all underwent ureteral reimplantation. Only one patient had f-UTI. In our study, in four patients with PICVUR after subureteric injection, we chose to perform redo-PIC cystography and subureteric injection instead of reimplantation. To our knowledge, this is the first paper to report redo-PIC cystography. Due to our success with redo-PIC cystography, and its decreased morbidity, mortality and cost plus increased patient and parent comfort, we advise this approach even in patients who continue to have f-UTI after PIC cystography.

Hagerty et al. ${ }^{14}$ concluded that PIC-VUR is clinically significant by determining that the incidence rate of f-UTI is lowered significantly by treatment of VUR identified by PIC cystography. We acknowledge that antireflux procedures do not eliminate the risk of infection. However, we found resolution of recurrent f-UTI in 39 patients $(95.1 \%)$.

It is plausible to consider that PIC cystography could artificially induce VUR in children. Edmondson et al. ${ }^{3}$ possibly thought that a hypotonic dyskinesia of the trigone musculature may account for the demonstration of PICVUR when the standard VCUG finding is normal. With an empty bladder and low trigone muscle tone, urine may reflux to the ureter. Yet this notion is refuted by Rubenstein et al. ${ }^{9}$ because reflux was not demonstrated in any child in the control group without a history of f-UTIs. Navai et al. ${ }^{15}$ measured the pressure at the ureteral orifice during PIC cystography was $17 \mathrm{cmH} 20$ by $8.5 \mathrm{~F}$ cystoscopy and decreased to $4 \mathrm{cmH} 20$ for $17.5 \mathrm{~F}$ cystoscopy. Another drawback of PIC cystography is the requirement for general anesthesia, but PIC cystography provides evaluation of the urethra, bladder and ureteral orifices. Once PIC-VUR is demonstrated ureteral injection can be performed in the same session. ${ }^{9}$ We believe the risk of general anesthesia is acceptable in order to treat patients with PIC-VUR, who are under the risk of renal scarring after each f-UTI.

In our study, we also performed preoperative renal ultrasonography and Tc-99m DMSA in our patients. Our data revealed that preoperative renal ultrasonography was abnormal in 8 
patients and preoperative Tc-99m DMSA was abnormal in 20 patients. Tareen et al. ${ }^{5}$ showed that four of five patients with VUR on PIC cystography had renal scarring or renal function loss. They concluded that if a child has a second infection that is confirmed to involve the upper tracts by DMSA scan, the next step in patients with negative VCUG should involve PIC cystography. A drawback of this study was the small number of patients. Berger et al. ${ }^{16}$ sought to identify the relationship between PIC-VUR and renal scarring in Tc-99m DMSA. The data showed that there was statistically significant association between PIC-VUR grade and severity of renal scarring. In our study, we found that USG and PIC cystography findings did not correlate. Similarly, while all patients with positive DMSA findings also had positive PIC-VUR, more importantly, a significant number of patients with no DMSA findings also had positive PIC-VUR. We therefore strongly advise that all patients, regardless of DMSA and USG findings, should undergo PIC cystography when f-UTI reoccurs despite negative VCUG. In a recent study, Pichler et al. ${ }^{17}$ reported good results when patients with recurrent UTI with negative VCUG but findings on DMSA were treated with subureteric injection for occult VUR.

Many children with recurrent f-UTIs do not demonstrate VUR on conventional VCUG. This may become associated with significant morbidity. Recurrent f-UTIs increase the risk of chronic kidney disease, hypertension and end stage renal disease. Renal scarring is implicated in long term sequelae. Therefore, guidelines for the management of f-UTI have advocated aggressive treatment and extensive imaging studies to detect VUR in targeted children.8,18 Current research on PIC cystography shows that the PIC cystography can identify clinically significant occult VUR. We suggest that PIC cystography should be part of the algorithm in evaluating and treating patients with recurrent f-UTI with no VUR on conventional VCUG.

The retrospective design and moderate sample size are the major limitations of our study. Another important drawback is that the majority of radiological studies of our patients were performed in other centres. The economical, social and health impact of performing redo renal USG, DMSA and VCUGs in these patients would not be acceptable and was therefore not performed.

The ultimate goal of treating children with $\mathrm{f}$-UTI is preventing long-term sequelae such as f-UTI recurrence, renal scarring, renal insufficiency, hypertension and end stage renal disease. In patients with recurrent f-UTI and no VUR on conventional VCUG, we strongly advise the use of PIC cystography, even when renal USG and DMSA are normal. We have found that this method allows for the treatment of 'occult' VUR, therefore potentially preventing further renal damage and morbidity. Our study also supports the existence of "occult" VUR. A large percent of patients treated with subureteric injection for PIC-VUR no longer had f-UTI. Also, those who continued to have f-UTI underwent a second redo-PIC cystography that demonstrated continuing VUR, that when treated with subureteric injection once again, resulted in the cessation of $\mathrm{f}$-UTI.

Although there are debates on PIC cystogram approach in recurrent f-UTIs in patients with occult VUR, our results in this study showed that PIC cystogram may be used in individual patients for clinical benefit. Further prospective and large sample sized studies are needed to confirm the results of this study.

\section{REFERENCES}

1. Rushton HG. The evaluation of acute pyelonephritis and renal scarring with technetium $99 \mathrm{~m}$-dimercaptosuccinic acid renal scintigraphy: evolving concepts and future directions. Pediatr Nephrol 1997; 11: 108-120.

2. Mir S, Ertan P, Ozkayin N. Risk factors for renal scarring in children with primary vesicoureteral reflux disease. Saudi J Kidney Dis Transpl 2013; 24: 54-59.

3. Edmondson JD, Maizels M, Alpert SA, et al. Multiinstitutional experience with PIC cystography--incidence of occult vesicoureteral reflux in children with febrile urinary tract infections. Urology 2006; 67: 608-611.

4. Elmore JM, Kirsch AJ, Lyles RH, Perez-Brayfield MR, Scherz HC. New contralateral vesicoureteral reflux following dextranomer/hyaluronic Acid implantation: incidence and identification of a high risk group. J Urol 2006; 175: 1097-1100; discussion 1100-1101.

5. Tareen BU, Bui D, McMahon DR, Nasrallah PF. Role of positional instillation of contrast cystography in the algorithm for evaluating children with confirmed pyelonephritis. Urology 2006; 67: 1055-1057; discussion 1058-1059.

6. Pinto KJ, Pugach J, Saalfield J. Lack of usefulness of positioned instillation of contrast cystogram after injection of dextranomer/hyaluronic acid. J Urol 2006; 176: 2654-2656. 
7. Noe HN, Williams MA. Clinical experience with positional installation of contrast cystography and simultaneous Deflux injection in children with occult vesicoureteral reflux. J Pediatr Urol 2007; 3: 375-377.

8. Paintsil E. Update on recent guidelines for the management of urinary tract infections in children: the shifting paradigm. Curr Opin Pediatr 2013; 25: 88-94.

9. Rubenstein JN, Maizels M, Kim SC, Houston JT. The PIC cystogram: a novel approach to identify "occult" vesicoureteral reflux in children with febrile urinary tract infections. J Urol 2003; 169: 2339-2343.

10. Nevéus T, von Gontard A, Hoebeke P, et al. The standardization of terminology of lower urinary tract function in children and adolescents: report from the Standardisation Committee of the International Children's Continence Society. J Urol 2006; 176: 314324.

11. National Collaborating Centre for Women's and Children's Health. Urinary tract infection in children: Diagnosis, treatment and long-term management. 2007; 54: 84-114.

12. Mingin GC, Hinds A, Nguyen HT, Baskin LS. Children with a febrile urinary tract infection and a negative radiologic workup: factors predictive of recurrence. Urology 2004; 63: 562-565; discussion 565.
13. Bratslavsky G, Feustel PJ, Aslan AR, Kogan BA. Recurrence risk in infants with urinary tract infections and a negative radiographic evaluation. J Urol 2004; 172: 1610-1613; discussion 1613.

14. Hagerty JA, Maizels M, Cheng EY. The PIC cystogram: its place in the treatment algorithm of recurrent febrile UTIs. Adv Urol 2008: 763620.

15. Navai N, Halperin W, Maizels M, Yerkes EB, Hagerty J, Kaplan WE. Demonstraing Vesicoureteral Reflux by Positioning the Instillation of Contrast (PIC) Cystography is a Physiological Test by Manometry. J Urol 2008; 179: 203-203.

16. Berger C, Becker T, Koen M, et al. Positioning irrigation of contrast cystography for diagnosis of occult vesicoureteric reflux: association with technetium- $99 \mathrm{~m}$ dimercaptosuccinic acid scans. J Pediatr Urol 2013; 9: 846-850.

17. Pichler R, Heidegger I, Schlenck B, Horninger W, Oswald J. Clinical outcome after endoscopic therapy for occult vesicoureteral reflux in females: preliminary results of a retrospective case series. Scand J Urol 2014; 48: 387-392.

18. Smellie JM, Barratt TM, Chantler C, et al. Medical versus surgical treatment in children with severe bilateral vesicoureteric reflux and bilateral nephropathy: a randomised trial. Lancet 2001; 357: 1329-1333. 